\title{
Integrated Flow Sensing for Focal Biochemical Stimulation
}

\author{
Li-Yuan Chang ${ }^{1}$, Po-Ying Li ${ }^{2}$, Lingyun Zhao ${ }^{3}$, Tuan Hoang ${ }^{1}$, and Ellis Meng ${ }^{1, *}$ \\ ${ }^{I}$ Department of Biomedical Engineering, University of Southern California, Los Angeles, CA, USA. \\ ${ }^{2}$ Department of Electrical Engineering, University of Southern California, Los Angeles, CA, USA. \\ ${ }^{3}$ Department of Electronics Engineering, Tsinghua University, Beijing, P.R. China.
}

\begin{abstract}
A microfluidic platform for focal stimulation of cells and tissue allows precise biochemical control of the extracellular microenvironment. This interface consists of a polymer microchannel having a single pore through which chemicals are selectively diffused or ejected. Towards the realization of realtime feedback control of focal biochemical stimulation, integrated thermal flow sensors were used to monitor fluid release. Delivery of fluorescent dye to rat retina is also presented.
\end{abstract}

Keywords-thermal flow sensor; Parylene C; microfluidic delivery; chemical stimulation

\section{INTRODUCTION}

Cells use chemical gradients for a number of critical functions, including cell signaling, nutrient supply, waste disposal, and gas exchange. There is great interest to manipulate the microenvironment and interface with cells. The conventional method for fluidic devices to release chemicals to cells is bulk fluid flow. This method does not provide targeted delivery to a select population of cells. Advancements in interfacing with individual cells, either in culture or within tissue, have focused on improving spatial resolution. A focused chemical gradient can isolate and target an individual cell to elicit a specific response and study its relationship within the cellular network or tissue organization. Compared to bulk flow or perfusion, release volume can be regulated to match physiologically relevant levels to the targeted cells. In addition, a localized release can minimize unintended biological responses or adverse consequences in the surrounding cells.

Microfluidic dosing devices have been introduced that enable selective stimulation of cells and study of cell membrane receptors and pathways [1-6]. Patterned laminar flows confined by polymer or glass microchannels demonstrate the microfluidic control of cell inactivation with cellular and sub-cellular resolution $[1,4,5]$. Cells cultured in a microchannel environment are selectively perfused with the desired solutions by control of laminar streams of flow. These devices are particularly suited for experiments on cell cultures but are difficult to implement in tissue samples. An alternative approach is to restrict chemical release from a small aperture (2-8 $\mu \mathrm{m}$ in diameter) $[2,3]$. This method is more amenable for producing localized chemical gradients and is compatible with focal delivery to tissues. Additional control of fluid release from an aperture was made possible by addressing each with an individual flow control channel [6]. However, integrated flow rate monitoring is still lacking.

This project was funded by the US NSF Early Career Award (Project code: EEC-0547544) and received summer student support from the Viterbi School of Engineering - Tsinghua University Visiting Scholars Program.

*Contact author: ellis.meng@usc.edu
A novel microfluidic platform was developed that was capable of controlled focal delivery of fluid from a "pore" to a cellular microenvironment in both passive (diffusion) and active (ejection) modes. Each pore is individually addressed with a dedicated microchannel. Furthermore, this focal chemical delivery platform is the first to feature integrated thermal flow sensors for monitoring flow rate of each microchannel and is thus capable of feedback control of focal delivery for each pore. Arrayed resistive sensors have previously been demonstrated to be simple to incorporate into MEMS fabrication processes, allow multiple modes of operation (hot-film, calorimetric, and time-of-flight), and can also be used for the determination of thermal properties of the working fluid [7-9]. The thermal sensors are capable of $\mathrm{nL} / \mathrm{min}$ range flow rate measurements. The design and fabrication of the microfluidic platform are discussed. Then thermal sensor calibration and demonstration of focal ex vivo fluid delivery to retinal flat mount are presented.

\section{DESIGN}

The building block of the microfluidic platform is a surface micromachined Parylene $\mathrm{C}$ microchannel with a single fluid delivery pore on a soda lime glass substrate (Fig. 1-2). The channel includes a linear array of integrated platinum thermal flow sensors arranged between the channel and substrate. Notably, this basic unit can be scaled up to large networks of microchannels with high pore density for selective stimulation of multiple sites. Focal delivery by a high-density array of individually controlled pores will enable sophisticated interfaces to complex biological tissues that allow new questions in biology to be investigated.

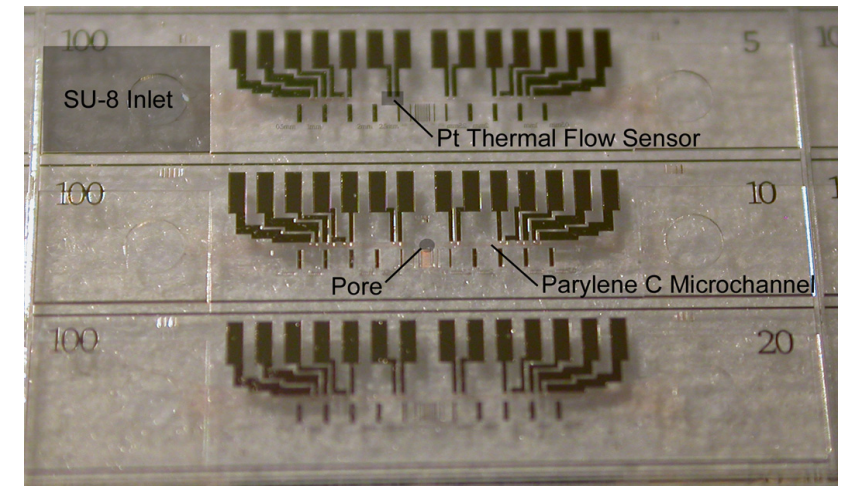

Figure 1. Image of fabricated microfluidic platform: die having three individual channels each having a different pore size indicated by the number on the upper right corner of each microchannel unit. 


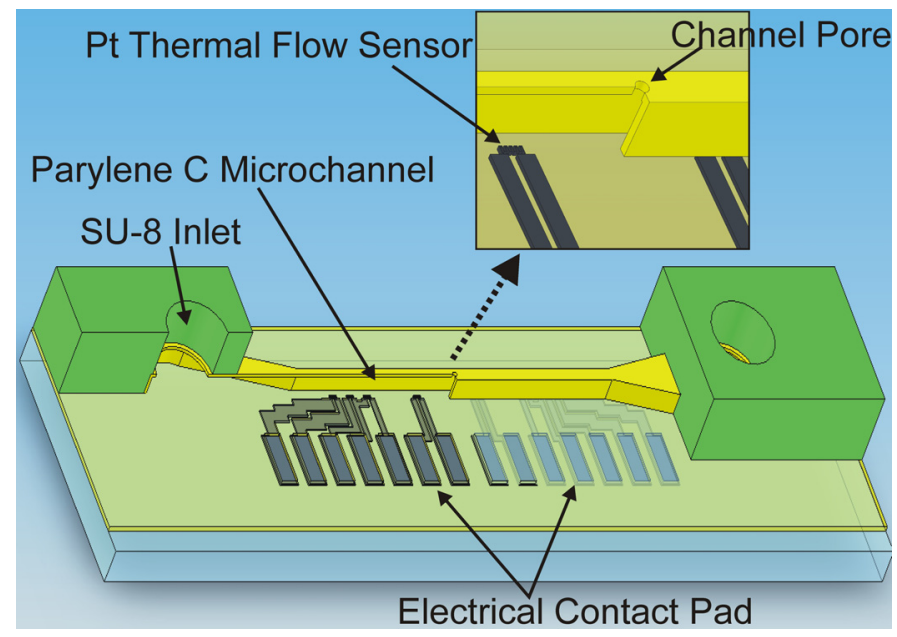

Figure 2. Three dimensional schematic detailing the features of the microfluidic platform. Figure not to scale.

SU-8 patterns surround the inlet/outlet ports of the microchannel and add structural support of the channel access points during packaging. The funnel connecting the access ports to the microchannel is supported with Parylene posts for increased mechanical strength. The microchannel dimensions are $6 \mathrm{~mm}$ x $100 \mu \mathrm{m}$ x $4 \mu \mathrm{m}(\mathrm{L} \mathrm{x} \mathrm{W} \mathrm{x} \mathrm{H)} \mathrm{with} 2 \mu \mathrm{m}$ thick walls and a single pore measuring 5,10 , or $20 \mu \mathrm{m}$ in diameter is located at the center of each channel to allow focal delivery to cells in the immediate vicinity of the pore. Thin film platinum thermal flow sensors are strategically arranged upstream and downstream of the pore so that fluid delivery through the pore can be calculated from the difference in measured flow rate before and after the pore. By utilizing an array of sensors, it is possible to use different sensing modes to determine fluid flow rate, direction, and to detect clogs [7,9].

Parylene $\mathrm{C}$ was selected as the channel and cell/tissue interface material for its excellent material properties, compatibility with micro- and nanofabrication processes, and its biocompatibility with both short- and long-term growth of cells/tissues [10-12]. Furthermore, Parylene C is transparent and allows convenient observation of channel contents by light and fluorescence microscopy.

Flow sensing based on thermal principles using Pt thin film resistors description was selected for ease of integration and implementation in the microfluidic platform. Furthermore, $\mathrm{Pt}$ is well known as a stable thermal sensor material. Thermal flow sensors are able to detect changes in sensor resistance resulting from heat transfer by forced convection from the heated sensing element to adjacent fluid flow.

\section{DEVICE FABRICATION}

A summary of the device fabrication process is described (Fig. 3). First, photoresist was applied to a 3" soda lime wafer and platinum sensors were patterned in preparation for lift-off (Fig. 3A). A $200 \AA$ titanium adhesion layer followed by a $2000 \AA$ thick platinum film were electron-beam evaporated. A standard lift-off process was performed to define the individual sensors (Fig. 3B). A $2 \mu \mathrm{m}$ thick layer of Parylene C was vapor-deposited over the entire wafer (Fig. 3C). Then microchannels were then defined by patterning a $4 \mu \mathrm{m}$ thick sacrificial photoresist layer (Fig. 3D). The sacrificial photoresist channel pattern also incorporated round holes for the formation of support posts along the channel length. A second layer of $2 \mu \mathrm{m}$ thick Parylene $\mathrm{C}$ was coated to complete the microchannels (Fig. 3E). The flow sensor contact pad openings, microchannel inlet/outlet ports, and fluid delivery pores were patterned and selectively etched using oxygen plasma reactive ion etching (Fig. 3F-3G) [13]. $75 \mu \mathrm{m}$ thick SU-8 inlet/outlet fluidic ports were photolithographically defined to provide structural support for the microchannels during packaging (Fig. 3H). Wafers were diced and individual dies were separated. Dies were immersed in isopropyl alcohol at room temperature over several days to gently remove the sacrificial photoresist in the channels.

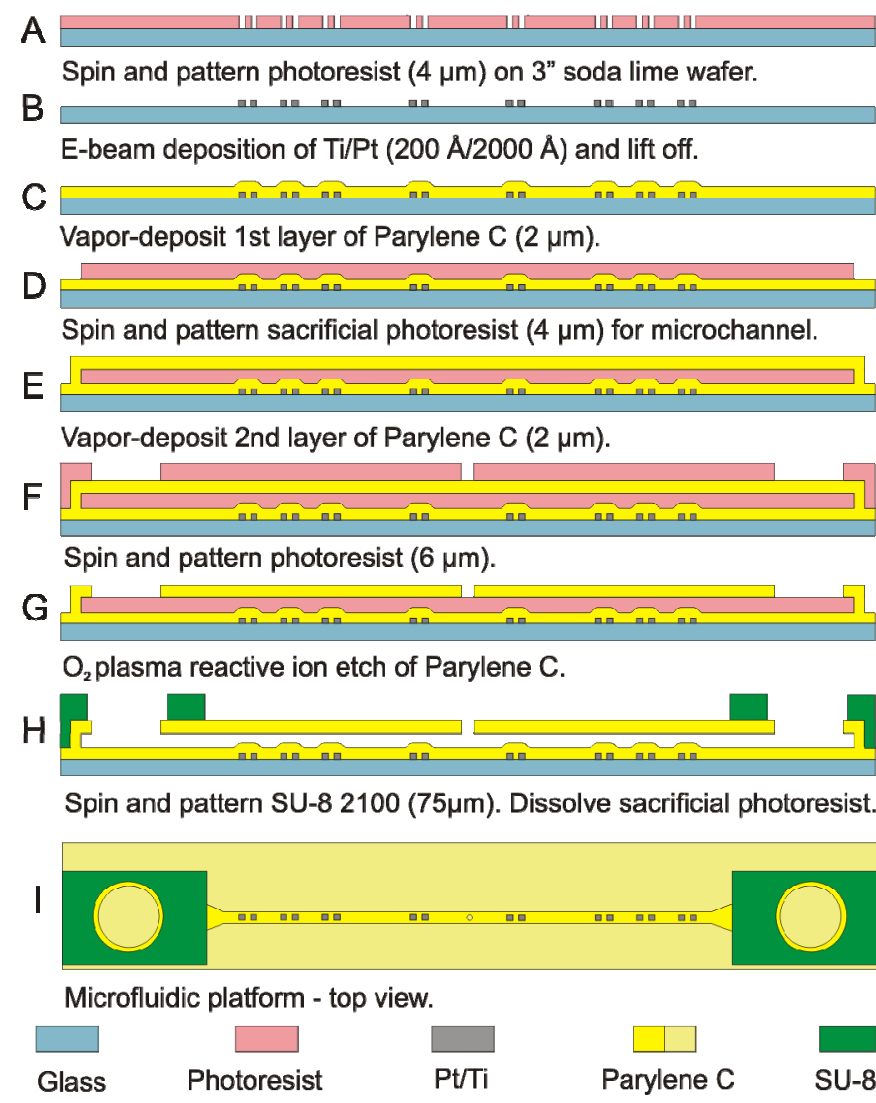

Figure 3. Simplified process flow of the microfluidic platform.

\section{PACKAGING}

Devices were packaged so as to enable simultaneous access to both electrical and fluidic connections (Fig. 4). First, devices were individually secured using adhesive to a printed circuit board (PCB). An electrical connection was established with the PCB via wire bonding and external wires were then soldered to larger contact pads on the PCB. A custom lasermachined acrylic clamping fixture with fluidic conduits was 
used to provide a convenient modular fluidic interface. The PCB to which the device is electrically connected was placed and manually-aligned between the two acrylic plates to establish a world-to-chip interface between the conduits in the acrylic and SU-8 access ports of the microfluidic device. Silicone rubber gaskets (Sylgard 184) were positioned between the acrylic clamp and device to provide adequate sealing. The packaged device was connected with conventional tubing to precision pressure-tight glass syringes (Hamilton Company) driven by a syringe pump (Harvard Apparatus). This package approach allowed for rapid device mounting and plumbing.

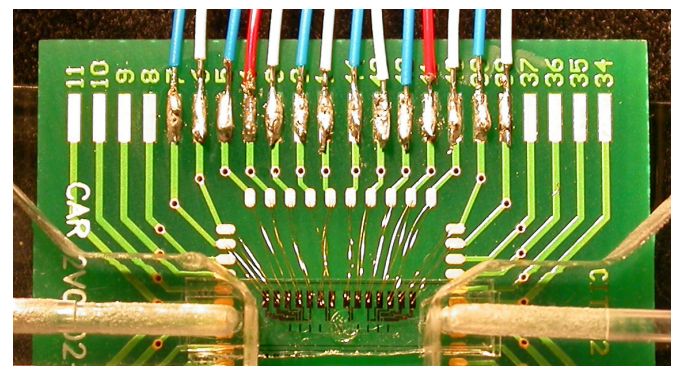

Figure 4. Single channel microfluidic platform mounted on and wire bonded to a PCB. The PCB assembly was clamped in an acrylic package to establish fluidic connections.

\section{RESUlts AND DiscUSSION}

\section{A. Bench Top Testing of Flow Sensors}

Calibration and flow sensing characterization experiments were performed in a thermally isolated and temperature controlled environmental chamber (Sun Electronic Systems) to minimize sensor output fluctuations due to ambient temperature variations. Controlled delivery of test fluids (deioinized water and fluorescein) were introduced to the packaged microfluidic platform using a syringe pump. Thermal flow sensors were operated in constant current mode (Agilent Technologies) and the sensor output was digitally acquired with a precision voltmeter (Keithley) controlled by a custom LabView program. The experimental setup is illustrated in Fig 5.

Thin film platinum thermal flow sensors were carefully calibrated and tested. The zero power resistance of the sensor was linearly extrapolated from values measured at two applied currents (Fig. 6).

The sensor temperature coefficient of resistivity (TCR) was obtained by calibration in a temperature controlled environmental chamber. Acquisition of the data was automated by using a custom LabView program to control the chamber temperature and acquire sensor resistance measurements. The average TCR obtained was $2.20 \times 10^{-3} /{ }^{\circ} \mathrm{C}$ and a typical TCR measurement is shown in Fig. 7.

The IV curve and the overheat behavior of the sensor were also collected (Figs. 8-9). As expected, the IV response was linear. To optimize sensor frequency response and sensitivity the overheat ratio should be maximized. However, for biological applications in which cells and tissue will be placed in close proximity to the sensors, a maximum overheat temperature of $10^{\circ} \mathrm{C}$ was selected to prevent adverse biological consequences resulting from unintentional heating.

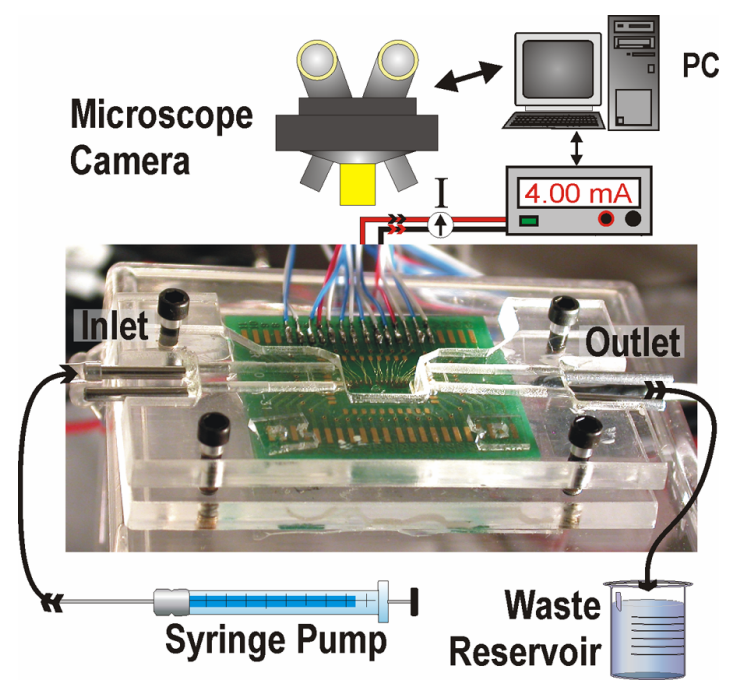

Figure 5. Microfluidic platform and platinum thermal flow sensor test setup.

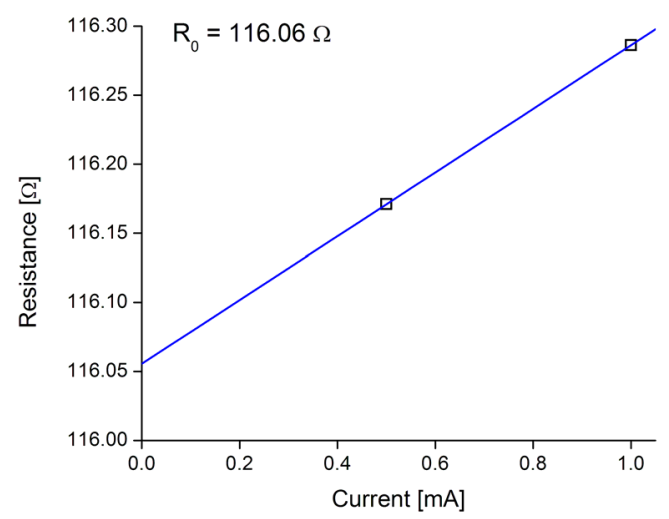

Figure 6. Platinum flow sensor zero power resistance.

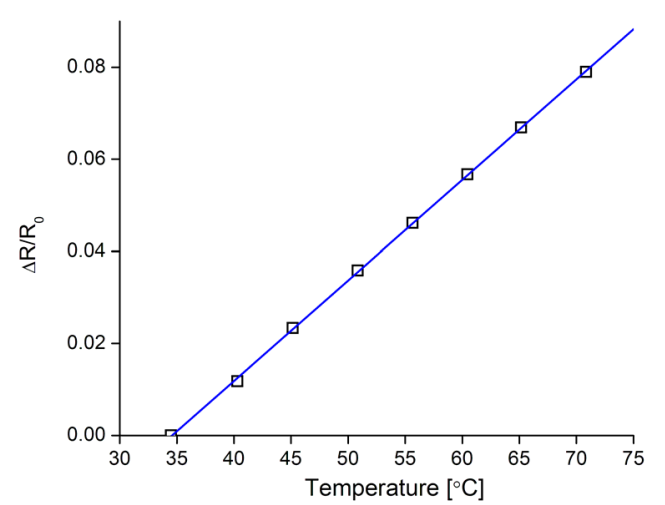

Figure 7. Platinum flow sensor TCR calibration data with a linear fit. 


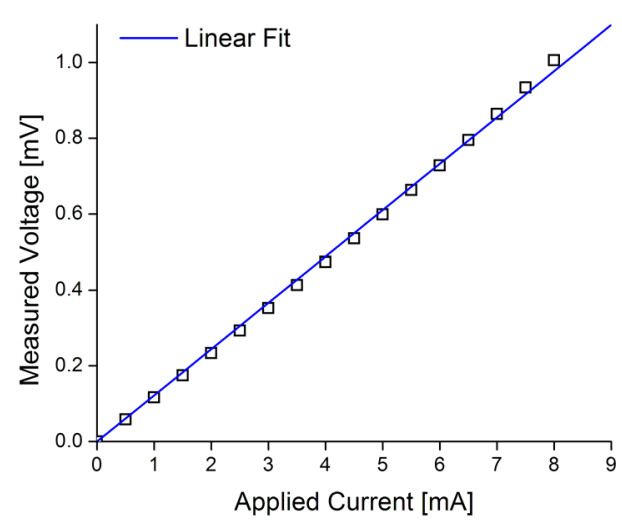

Figure 8. Representative platinum flow sensor IV curve.

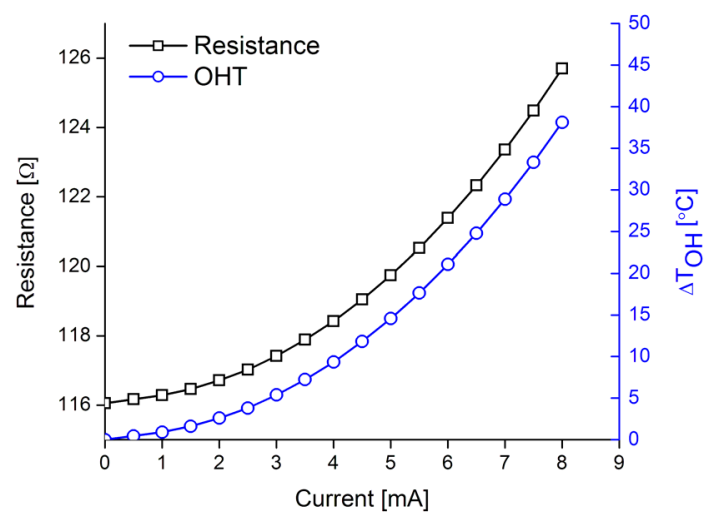

Figure 9. Platinum flow sensor overheat behavior.

Flow rate sensing was performed with the fluid delivery pore sealed off by epoxy to create a closed channel. Flow rate data for both deionized (DI) water and fluorescein $(0.11 \mathrm{mM})$ were obtained by operating the sensor in hot film mode under constant current biasing at $4 \mathrm{~mA}$ (Fig. 10).

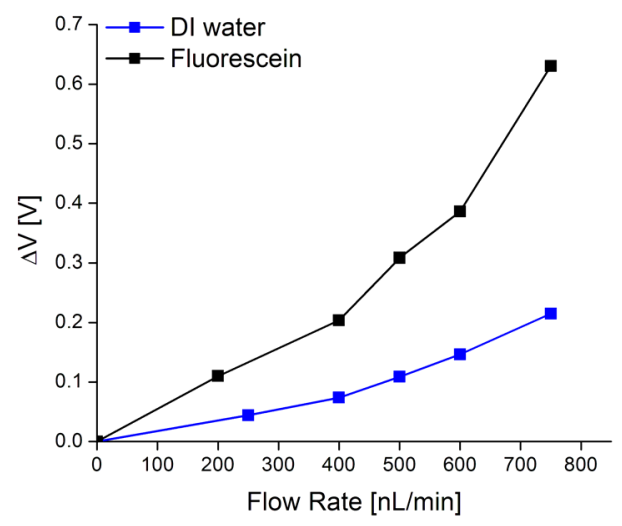

Figure 10. Hot film response for $4 \mathrm{~mA}$ constant current biasing. Both DI water and fluorscein responses were obtained.
It was observed that thin $2 \mu \mathrm{m}$ thick Parylene $\mathrm{C}$ wall at the entrance to the microchannel (funnel-shaped) bulged with increasing input flow rate (Fig. 11a). It is suspected that at higher flow rates, the increased fluid driving pressure induced strain and ultimately plastic deformation of the polymer channel wall. The bulging entrance funnel created room for fluid accumulation. In the absence of an external counter pressure or structural support to prevent wall deformation, the actual flow rates in the microchannel are likely to be lower than the input flow rates set by the syringe pump. To accurately measure flow rate in the microchannel, the SU-8 support and entrance funnel designs were modified to circumvent plastic deformation and eliminate fluid accumulation in the entrance funnel (Fig. 11b).

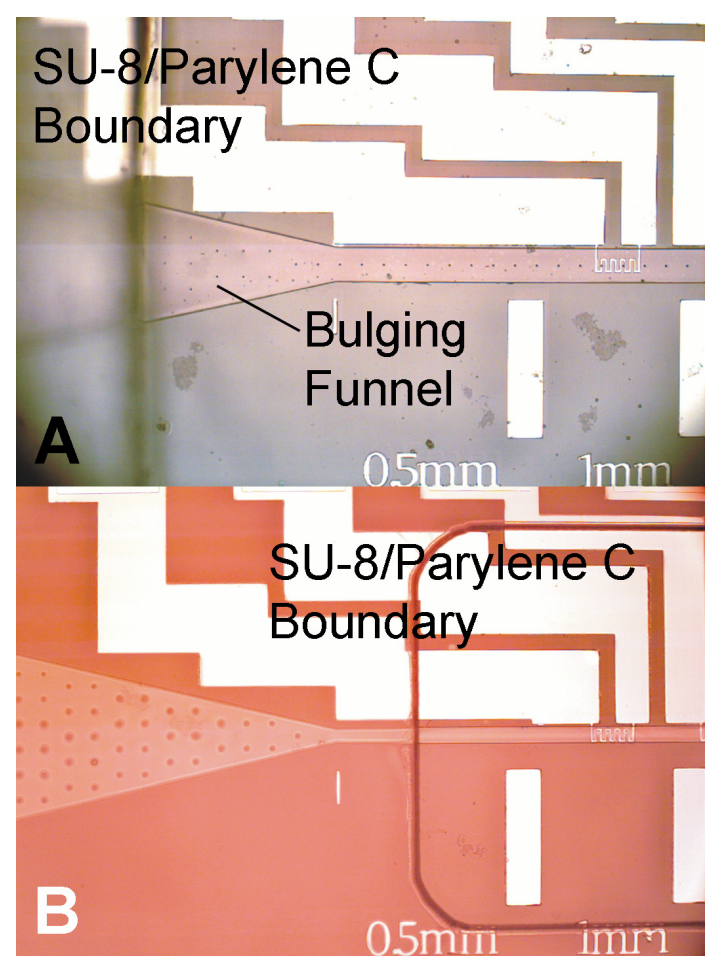

Figure 11. (a) Microchannel access port partially supported by an SU-8 protective layer. Bulging of the funnel area is observed for increased fluid driving pressures. (b) An improved access port with extended SU-8 protective layer covering the entire entrance funnel. The SU- 8 also forms a fluid reservoir around the delivery pore.

\section{B. Bench Top Testing of Fluid Delivery Through Pore}

Delivery of rhodamine B $(0.11 \mathrm{mM})$ through the pore by diffusion and syringe pump driven ejection was also characterized. In the diffusion studies (passive mode), channels were filled with rhodamine $\mathrm{B}$ and the dye was allowed to diffuse through the pore into the DI water bath above. Fluorescence microscopy was used to collect timelapse images of the diffusion process. Images were analyzed using Image $\mathrm{J}(\mathrm{NIH})$ and the radius of the diffusion plume was determined with the aid of a calibrated scale. It was determined that radius of the fluorescent plume increased at an average diffusion rate of $4.9 \mu \mathrm{m} / \mathrm{s}$ (10 $\mu \mathrm{m}$ pore) and the 
normalized optical intensity of the plume increased respectively (Fig. 12).

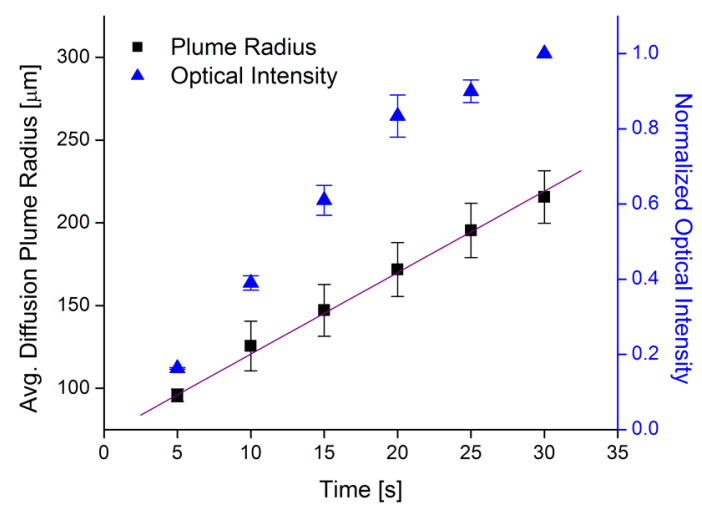

Figure 12. Diffusion radius of rhodamine B delivered through pore with a linear fit on the radius data (mean $\pm \mathrm{SE}, \mathrm{n}=3$ ).

Measured diffusion optical intensity profiles (onedimension) show correspondence to the theoretical constant point source diffusion concentration profiles (Fig. 13). The point source diffusion model is given below:

$$
\begin{aligned}
& u(t, x)=C_{1}[1-\operatorname{erf}(x / \sqrt{4 D t})] \\
& \frac{\delta u}{\delta t}=D \frac{\partial^{2} u}{\partial x^{2}}, t>0, x \in(0, \infty), \\
& u(t, 0)=C_{1}, \lim _{x \rightarrow \infty} u(t, x)=0, \\
& u(0, x)=0, x \in(0, \infty)
\end{aligned}
$$

where $u$ is concentration, $D$ is the diffusion coefficient, $x$ is the distance from the aperture, and $C_{1}$ is the initial concentration which was set to 1 .

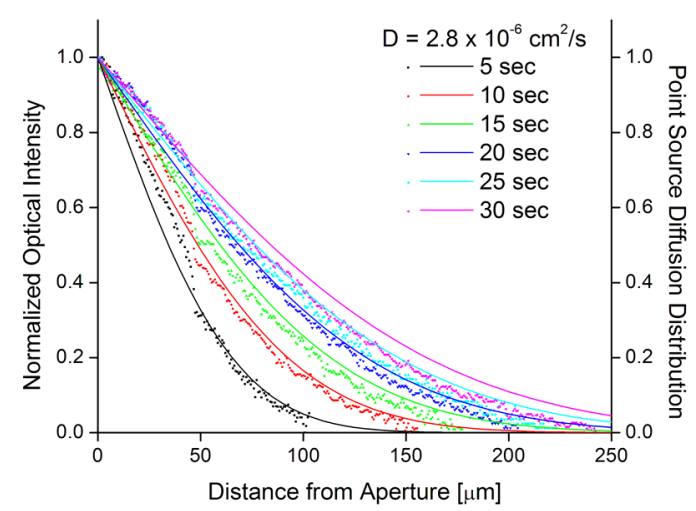

Figure 13. Measured diffusion of rhodamine B (dots) compared to point source diffusion theory (solid lines) $\left(D=2.8 \times 10^{-6} \mathrm{~cm}^{2} / \mathrm{s}\right.$ in $20^{\circ} \mathrm{C}$ solution [14]).
Fluid ejection (active mode) of dispensed dye through the pore into air was monitored with time-lapse light microscopy images. The radius of the observed dye droplet was measured with the aid of ImageJ software and the corresponding volume was calculated with an estimated hemispherical geometry. The flow rate was then calculated from the accumulated volume over time and subsequently corrected for evaporation (Fig. 14). With 50 to $750 \mathrm{~nL} / \mathrm{min}$ input flow from the syringe pump, the ejection flow rate from the $10 \mu \mathrm{m}$ diameter pore ranged from 16 to $106 \mathrm{~nL} / \mathrm{min}$. This demonstrates localized delivery of $\mathrm{pL}-\mathrm{nL}$ volumes within a controlled sphere of influence directly above the pore.

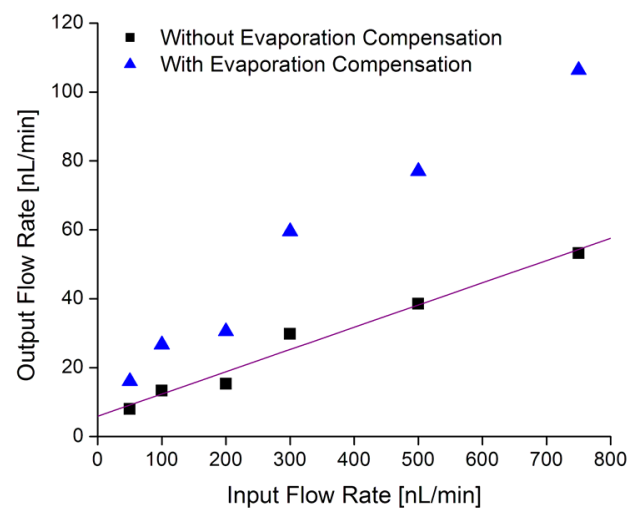

Figure 14. Pressure driven ejection flow delivery of rhodamine B through pore into air with and without evaporation correction. A linear fit was applied to the raw, uncompensated data.

\section{Tissue Study}

Preliminary demonstration of in vitro focal delivery to dissected wildtype Sprague-Dawley rat retinal tissue was demonstrated. A retinal flat mount was positioned over the microfluidic delivery platform and held in place using a ring of Whatman filter paper and a metallic ring clamp (Fig 15a-b). The tissue was focally perfused with rhodamine $\mathrm{B}$ via the pore and then removed for visual inspection. Both focal diffusion and ejection were recorded by time-lapsed photography $(0.25$ second intervals) under fluorescence microscopy. First, diffusion-based delivery was performed over a 25 second period. Next, ejection mode was initiated by priming for one minute. Finally, ejection-based delivery was captured over a 25 second period. The time course of diffusion and ejection at select times are shown in Fig. 15c and 15d, respectively. Dye accumulation in the retina can be visually observed directly above the pore. By visual inspection, the rate of dye accumulation in the retina appears to be more dramatic in ejection mode compared to diffusion mode. 


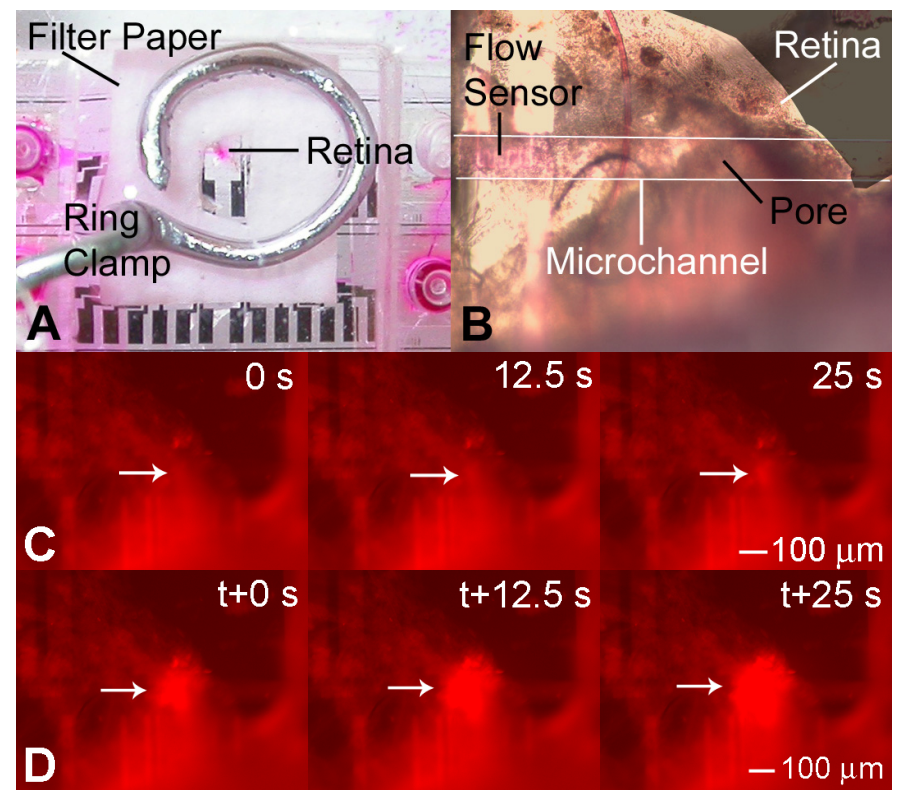

Figure 15. (a) Experimental test setup for rhodamine B focal delivery to rat retina, (b) brightfield image of retina mounted over microchannel and pore, (c) fluoresence time-lapse images of focal delivery of rhodamine B by diffusion to rat retina, and (d) ejection mode time-lapse images focal delivery of rhodamine B. The arrow indicates the vicinity above the $20 \mu \mathrm{m}$ pore.

\section{CONCLUSION}

Microfluidic platform for controlled and focal delivery of soluble agents to cells and tissue has been demonstrated. The platform consists of a small diameter delivery pore at the center addressed by a dedicated Parylene $\mathrm{C}$ microchannel. Each channel features integrated flow sensing in the form of a Pt thermal sensor array. Characterization of the flow sensors and fluid delivery capability of platform under diffusion and ejection modes was performed. Preliminary demonstration of in vitro focal delivery to rat retina was achieved in both diffusion and ejection modes. Future work includes correlation of syringe pump driven flow with flow sensor response. This will enable feedback control of focal delivery. Focal delivery by a high-density array of individually controlled pores will enable sophisticated interfaces to complex biological tissues that allow new questions in biology to be investigated.

\section{ACKNOWLEDGMENT}

The authors would like to thank Mr. Merrill Roragen and Dr. Donghai Zhu for assistance with fabrication; Dr. David Merwine for providing the retinal tissue; and members of the Biomedical Microsystem Lab at the University of Southern California for their assistance.

\section{REFERENCES}

[1] H. Kaji, M. Nishizawa, and T. Matsue, "Localized chemical stimulation to micropatterned cells using multiple laminar fluid flows," Lab on a Chip, vol. 3, pp. 208-211, 2003.

[2] T. F. Kosar, A. Tourovskaia, X. Figueroa-Masot, M. E. Adams, and A. Folch, "A nanofabricated planar aperture as a mimic of the nerve-muscle contact during synaptogenesis," Lab on a Chip, vol. 6, pp. 632-638, 2006.

[3] M. C. Peterman, J. Noolandi, M. S. Blumenkranz, and H. A. Fishman, "Localized chemical release from an artificial synapse chip," Proceedings of the National Academy of Sciences of the United States of America, vol. 101, pp. 9951-9954, 2004.

[4] S. Takayama, E. Ostuni, P. LeDuc, K. Naruse, D. E. Ingber, and G. M. Whitesides, "Selective chemical treatment of cellular microdomains using multiple laminar streams," Chemistry \& Biology, vol. 10, pp. 123130, 2003.

[5] A. Tourovskaia, T. F. Kosar, and A. Folch, "Local induction of acetylcholine receptor clustering in myotube cultures using microfluidic application of agrin," Biophysical Journal, vol. 90, pp. 2192-2198, 2006.

[6] N. Z. Mehenti, H. A. Fishman, and S. F. Bent, "A model neural interface based on functional chemical stimulation," Biomedical Microdevices, vol. 9 , pp. 579-586, 2007.

[7] E. Meng and Y.-C. Tai, "A Parylene MEMS Flow Sensing Array," in Transducers 2003. Boston, MA, 2003, pp. 686-689.

[8] Q. Lin, F. K. Jiang, X. Q. Wang, Y. Xu, Z. G. Han, Y. C. Tai, J. Lew, and C. M. Ho, "Experiments and simulations of MEMS thermal sensors for wall shear-stress measurements in aerodynamic control applications," Journal of Micromechanics and Microengineering, vol. 14, pp. 1640-1649, 2004.

[9] E. Meng, P.-Y. Li, and Y. C. Tai, "A Biocompatible Parylene Flow Sensing Array," unpublished.

[10] J. I. Kroschwitz, Kirk-Othmer Encyclopedia of Chemical Technology. New York: John Wiley \& Sons, Inc., 1998.

[11] J. Lahann, "Vapor-based polymer coatings for potential biomedical applications," Polymer International, vol. 55, pp. 1361-1370, 2006.

[12] B. A. Weisenberg and D. L. Mooradian, "Hemocompatibility of materials used in microelectromechanical systems: Platelet adhesion and morphology in vitro," Journal of Biomedical Materials Research, vol. 60, pp. 283-291, 2002.

[13] E. Meng and Y.-C. Tai, "Parylene Etching Techniques for Microfluidics and BioMEMS," in MEMS 2005. Miami, Florida, 2005, pp. 568-71.

[14] K. Chattopadhyay, S. Saffarian, E. L. Elson, and C. Frieden, "Measurement of microsecond dynamic motion in the intestinal fatty acid binding protein by using fluorescence correlation spectroscopy," Proceedings of the National Academy of Sciences of the United States of America, vol. 99, pp. 14171-14176, 2002. 